GURU GOBIND SINGH

INDRAPRASTHA UNIVERSITY

\section{Box-Jenkins ARIMA Modelling: \\ Forecasting FDI in India}

Deepanshu Sharma, Kritika Phulli;

ASET, Guru Gobind Singh Indraprastha University

\section{ABSTRACT}

In the rapidly advancing dynamics of the economy trends of countries, the forecasting econometric techniques hold significant importance in the field of advance economics and management. Thus, this study intends to create Box Jenkins time series ARIMA model for analysing and predicting the trend of net FDI (Foreign Direct Investment) in India. The model was generated on the dataset of FDI inflow of India from the year 1950 to 2020 . The trend was analysed for the generation of the model that best fitted the forecasting. The study highlights the minimum AIC value and involves ADF test (Augmented Dickey-Fuller) to transform FDI data into stationary form for model generation. It proposes ARIMA $(1,1,4)$ model for optimal forecasting of net FDI inflow in India with an accuracy of $96.5 \%$. The model thus predicts the steadystate exponential growth of FDI inflow in the coming 2020-25.

\section{CONTACT}

Deepanshu Sharma,

Email : sharma.deepanshu97@gmail.com Phone:(+91) 7982100493

\section{Kritika Phulli}

Email: kritika1298phulli@gmail.com

Phone: (+91) 8800115306

\section{INTRODUCTION}

India is a rapidly developing country which is often seen as an investment ground by the industry giants of foreign countries. The recent trends show an exponential increase in the net foreign direct investment in India from the year 2010-2020 [1]. Thus, it becomes crucial for the policy makers and the economist to forecast the future inflows of investment in order to produce effective policies and take better decisions. The result of the better policies would help in overcoming the unbalanced market viability. ARIMA modelling is a technique used in statistics and econometrics which harness the advantages of both Auto Regression (AR) and Moving Average (MA) models by integrating them together to form auto regression integrated moving average model. Researchers like Perera and Prasanna utilized this model and customized it to forecast the FDI inflow in SAARC countries. [2] Nyoni et.al. used Box-Jenkins ARIMA for forecasting the FDI in Zimbabwe. [3]

This study aims at generating a customised Box-Jenkins ARIMA model for forecasting and analysing the trend of FDI in India. It also aims at evaluating the minimum AIC value for determining the fitness of the model and highlights the $95 \%$ confidence interval for the predictions made for year 2020-2025.

\section{METHODS AND MATERIALS}

The study is performed on the dataset of net FDI in India from 1950-2020(expressed as US dollars in millions). A customised box-Jenkins ARIMA model was generated in order to forecast the FDI and analysing the trend. Initially, the trend of the investment from 1950-2020 was visualised and it was found to be overall exponential in nature. While forecasting using ARIMA model, Stationarity and differencing has to be taken into consideration. Once the conditions are satisfied and validated using Augmented Dicker Fuller test, the ARIMA model was generated using the 3 parameters: $p, q$ and $d$.

The major steps followed are:

Step1: Model Identification - Augmented Dickey Fuller test used.

Step2: Parameter Estimation - Auto correlation Function (ACF) and Partial Auto Correlation Function (PACF) for identification of the order of ARIMA model (parameters $\mathrm{p}$ and $\mathrm{q}$ )

Step3: Training and Testing the model- Dividing the dataset into 7:3, the model is fitted using $\operatorname{ARIMA}(p, d, q)$. These model parameters were acquired using the minimum AIC score for best accuracy.

Step4: Diagnostic Check: Determining the Residuals of the ACF and PACF graphs for identical and independent distribution.

Step5: Forecasting the data: The future FDI expenditure was forecasted for forthcoming years along with its lower bound and upper bound values with $95 \%$ confidence.
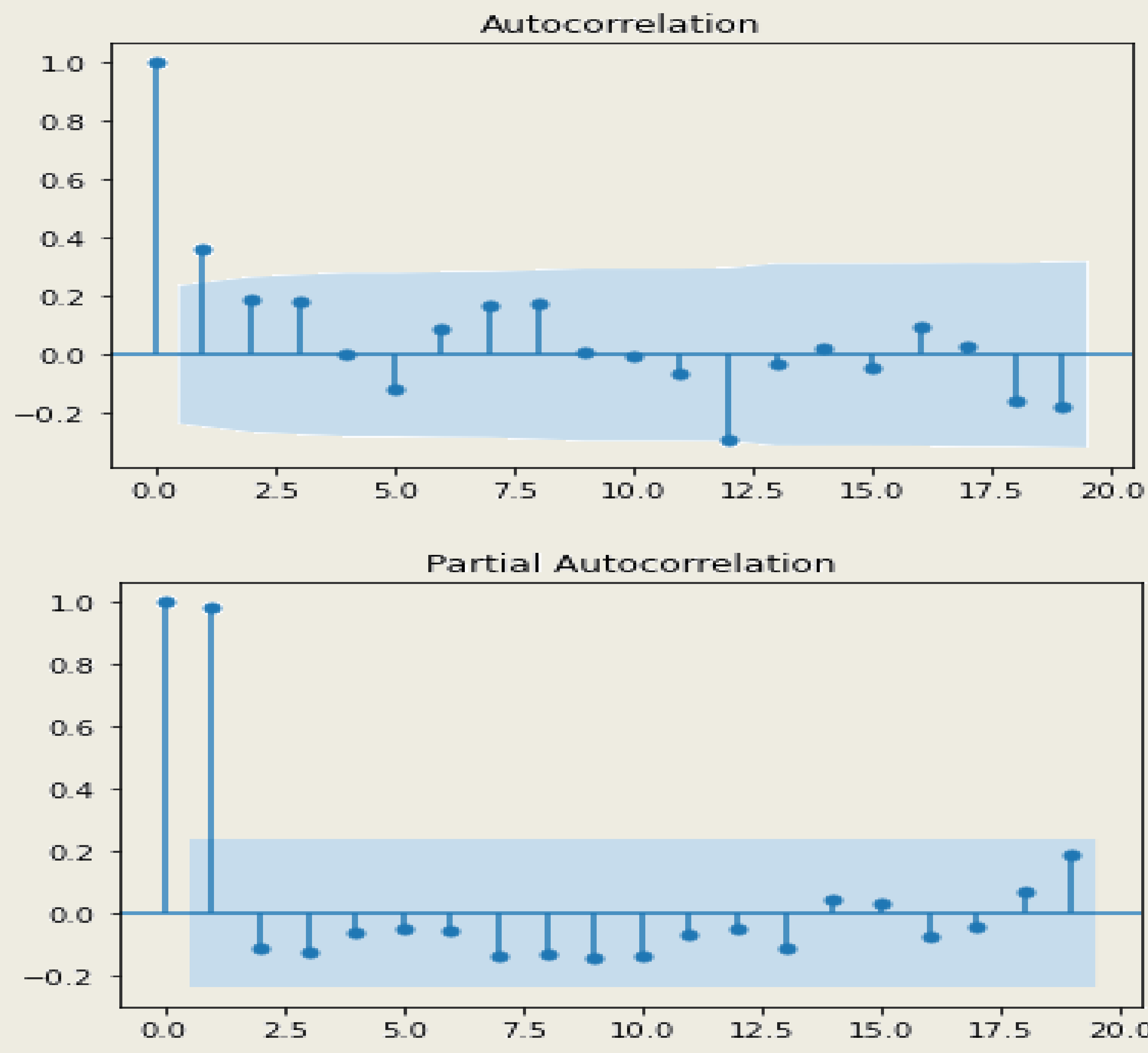

Fig 1. PACF \& ACF plot for $p$, q value determination

Table 1. Forecasted FDI in US million $\$$ with $95 \%$ interval values.

\begin{tabular}{|cccc|}
\hline Year & $\begin{array}{c}\text { Forecasted } \\
\text { (US million \$) }\end{array}$ & $\begin{array}{c}\text { Lower Bound } \\
\text { (US million } \mathbf{\text { ) }}\end{array}$ & $\begin{array}{c}\text { Upper Bound } \\
\text { (US million \$) }\end{array}$ \\
\hline $2020-21$ & 511095.16 & 36262.47 & 720354.3 \\
\hline $2021-22$ & 553191.54 & 33355.16 & 917461.7 \\
\hline $2022-23$ & 597142.74 & 30911.23 & 1153559 \\
\hline $2023-24$ & 645615.47 & 28887.26 & 1442917 \\
\hline $2024-25$ & 697363.89 & 27158.11 & 1790685 \\
\hline
\end{tabular}

\section{RESULTS \& DISCUSSION}

This section summarizes the findings of the research. The first and foremost outcomes depicts the trend of the time series data. Fig. 2. illustrates an exponential increasing trend of FDI in India.

The data was observed and transformed into its stationary form for modelling. For this, the dataset was pre-processed using the log transformation and differencing value (shift=1) and was validated using ADF test. Before transformation of data, ADF failed but after log transformation, ADF test accepted the null hypothesis, approximating to 0.0001 . The results of ADF test are reflected in Table 1.

Using the differencing value $(d=1)$ where the data is validated to be stationary, the other parameters: $p$ and $q$, are estimated using the ACF and PACF graphs. The graphs are depicted in the fig. 1.

The dataset was then used for training and testing purposes, divided into the ratio of 7:3 for generated the ARIMA model. A range of $p$ and $q$ values were used for modelling to achieve the stability of the generated model. For the range of $p$ and $q$ values, a respective AIC score is computed. The minimum AIC score was considered to select the optimal $p$ and $q$ values for further evaluations. Parameters obtained for optimal modelling of the time series data using ARIMA method are as follows:

$$
\text { p: } 1 ; d: 1 \text { q: 4; }
$$

The AIC value came out to be -13.03 , which is minimum among the others.

It is worth mentioning that the actual and predicted values of testing data comes out to be approximately close to each other with an accuracy of $96.4 \%$. The plot is described in fig. 2. After the model generation for ARIMA $(1,1,4)$, the forecasted values for the forthcoming years (2020-2025) are illustrated in the Table 1.

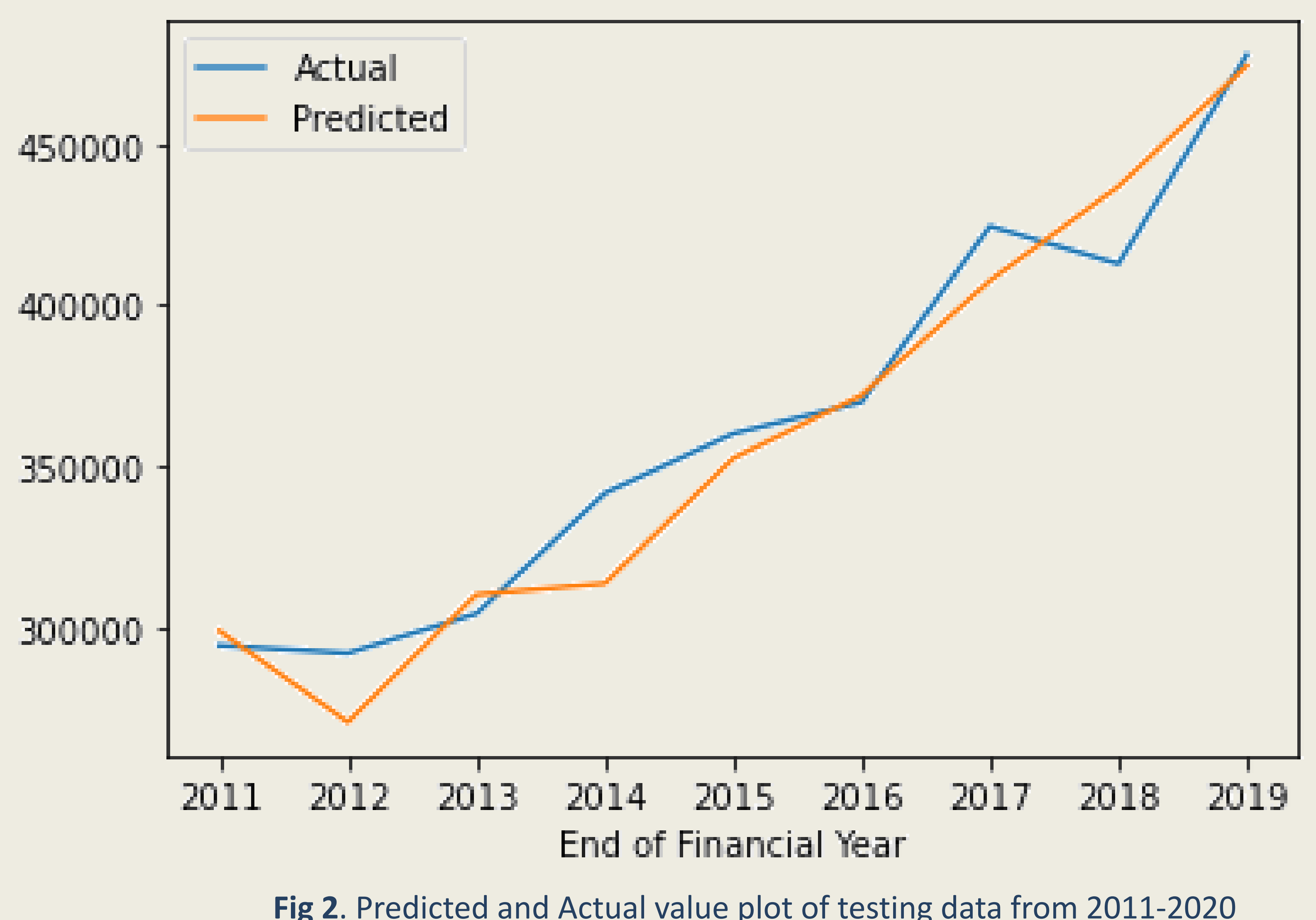

\section{CONCLUSIONS}

The rapid development of India is often observed as an investment opportunity by the huge industries of foreign countries. It has become necessary for the economist of India to forecast the future inflows of investments to structure the policies for better decision making. The proposed ARIMA model attains an accuracy of $96.4 \%$ with an increasing trend and a differencing value of 1 . The estimated parameters for auto regressive (AR) and moving average (MA) are $\mathrm{p}=1$ and $\mathrm{q}=4$ respectively using the PACF and ACF plotted graphs. The AIC score acquired accounts to -13.03 with the above formulated parameters, describing a better fitted ARIMA model when compared to other values of the parameters. This proposed model not only helps to forecast the Foreign Direct Investment for the forthcoming years but would also help in allocation of the designing the policies which in turn helps in overcoming the unbalanced market viability.

\section{REFERENCES}
Mani, $U$.
2. Perera, Prasanna. "Modeling and Forecasting Foreign Direct Investment (FDI) into SAARC for the
(nternational Journal of Business and Social Science 6.2
3. Nyoni, Thabani. "Box-Jenkins ARIMA approach to predicting net FDI inflows in Zimbabwe." (2018) 